\title{
First identification and genotyping of Enterocytozoon bieneusi and Encephalitozoon spp. in pet rabbits in China
}

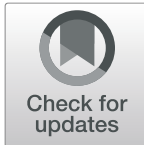

Lei Deng ${ }^{\dagger}$, Yijun Chai ${ }^{\dagger}$, Leiqiong Xiang ${ }^{\dagger}$, Wuyou Wang ${ }^{\dagger}$, Ziyao Zhou, Haifeng Liu, Zhijun Zhong, Hualin Fu and Guangneng Peng*

\begin{abstract}
Background: Microsporidia are common opportunistic parasites in humans and animals, including rabbits. However, only limited epidemiology data concern about the prevalence and molecular characterization of Enterocytozoon bieneusi and Encephalitozoon spp. in rabbits. This study is the first detection and genotyping of Microsporidia in pet rabbits in China.

Results: A total of 584 faecal specimens were collected from rabbits in pet shops from four cities in Sichuan province, China. The overall prevalence of microsporidia infection was $24.8 \%$ by nested PCR targeting the internal transcribed spacer (ITS) region of E. bieneusi and Encephalitozoon spp. respectively. E. bieneusi was the most common species $(n=$ 90, 15.4\%), followed by Encephalitozoon cuniculi $(n=34,5.8 \%)$ and Encephalitozoon intestinalis $(n=16,2.7 \%)$. Mixed infections (E. bieneusi and E. cuniculi) were detected in five another rabbits (0.9\%). Statistically significant differences in the prevalence of microsporidia were observed among different cities $\left(X^{2}=38.376, d f=3, P<0.01\right)$ and the rabbits older than 1 year were more likely to harbour microsporidia infections $\left(X^{2}=9.018, \mathrm{df}=2, P<0.05\right)$. Eleven distinct genotypes of E. bieneusi were obtained, including five known (SCO2, I, N, J, CHY1) and six novel genotypes (SCR01, SCR02, SCR04 to SCR07). SC02 was the most prevalent genotype in all tested cities (43.3\%, 39/90). Phylogenetic analysis showed that these genotypes were clustered into group 1-3 and group 10. Meanwhile, two genotypes (I and II) were identified by sequence analysis of the ITS region of E. cuniculi.

Conclusion: To the best of our knowledge, this is the first report of microsporidia infection in pet rabbits in China. Genotype SC02 and four novel genotypes were classified into potential zoonotic group 1, suggesting that pet rabbits may cause microsporidiosis in humans through zoonotic transmissions. These findings provide preliminary reference data for monitoring microsporidia infections in pet rabbits and humans.
\end{abstract}

Keywords: Microsporidia, Rabbits, ITS, Microsporidiosis, China

\footnotetext{
* Correspondence: pgn.sicau@163.com

'Lei Deng, Yijun Chai, Leiqiong Xiang and Wuyou Wang contributed equally to this work.

The Key Laboratory of Animal Disease and Human Health of Sichuan Province, College of Veterinary Medicine, Sichuan Agricultural University, Chengdu 611130, Sichuan, China
}

(c) The Author(s). 2020 Open Access This article is licensed under a Creative Commons Attribution 4.0 International License, which permits use, sharing, adaptation, distribution and reproduction in any medium or format, as long as you give appropriate credit to the original author(s) and the source, provide a link to the Creative Commons licence, and indicate if changes were made. The images or other third party material in this article are included in the article's Creative Commons licence, unless indicated otherwise in a credit line to the material. If material is not included in the article's Creative Commons licence and your intended use is not permitted by statutory regulation or exceeds the permitted use, you will need to obtain permission directly from the copyright holder. To view a copy of this licence, visit http://creativecommons.org/licenses/by/4.0/ The Creative Commons Public Domain Dedication waiver (http://creativecommons.org/publicdomain/zero/1.0/) applies to the data made available in this article, unless otherwise stated in a credit line to the data. 


\section{Background}

Microsporidia, as obligate intracellular parasites and classified as fungi, are emerging opportunistic pathogens that can infect many invertebrates and vertebrates, including humans and rabbits [1]. To date, the phylum Microsporidia consists of at least 200 genera and 1500 species, of which 17 microsporidia species have been detected in humans [2, 3]. Among them, Enterocytozoon bieneusi and Encephalitozoon spp. (including E. cuniculi, E. hellem, and E. intestinalis) are the four most common microsporidia species that infect humans, domestic animals, and wildlife $[4,5]$. Microsporidia are often considered as a major pathogen of chronic diarrhea in severely immunecompromised patients, such as AIDS patients and solid organ transplant recipients [6]. Besides, the discovery of microsporidia in water sources intended for human consumption has made it a Category B Priority Pathogen listed by the National Institutes of Health (NIH), and it has also been listed by the United States Environmental Protection Agency (EPA) as a microbial pollutant potentially causing waterborne outbreaks $[1,7]$.

More than $470 \mathrm{E}$. bieneusi genotypes have been identified in humans and animals based on sequence analysis of the internal transcribed spacer (ITS) region of ribosomal RNA (rRNA), with more new genotypes continually being found [8]. Some of these genotypes are considered to be host-specific, while others have zoonotic potential (e.g., SC02, D, EbpC, J, I and Type IV) [9]. Based on the number of $5^{\prime}$-GTTT-3' repeats in the ITS sequence, four distinct genotypes (genotype I -IV) of E. cuniculi have been identified [10,11]. E. hellem also has four genotypes (1 to 4 ) by analysis of the ITS sequence [12]. However, no intraspecific variation in the ITS sequence of $E$. intestinalis has been detected thus far.

In China, E. bieneusi and Encephalitozoon spp. have been identified in a broad range of wild and domestic animal hosts, including mammals, reptiles, and birds
[13]. Rabbits have been reported to harbor various zoonotic species (e.g., Cryptosporidium, Giardia, Microsporidia, and Toxoplasma gondii) and are considered to be a potential source of human infections $[14,15]$. However, only limited information is available on E. bieneusi and Encephalitozoon spp. infection in pet rabbits in China. Moreover, pet rabbits are popular companions and their close relationship with humans may represent a still not completely understood health threat. Therefore, the purpose of the present study was to determine the prevalence and molecular characteristics of microsporidia in faecal samples of pet rabbits, as well as to assess the zoonotic potential of these pathogens.

\section{Results}

Prevalence of E. bieneusi and Encephalitozoon spp.

A total of 584 faecal samples of pet rabbits from 12 pet shops in four cities in Sichuan province of southwestern of China were examined using molecular methods. The specific primers for E. bieneusi and Encephalitozoon spp. were used to determine the presence of microsporidia. In total, $24.8 \%(145 / 584)$ of the rabbits were found infected with microsporidians. Single-species infection was detected in 90 rabbits (15.4\%) for E. bieneusi. 34 (5.8\%) and $16(2.7 \%)$ E. cuniculi and E. intestinalis monoinfections were identified respectively (Table 1). Encephalitozoon hellem was not identified in the surveyed population. In addition, 5 samples were identified as coinfection (0.9\%) (Table 1$)$.

Microsporidia-positive samples were detected in all tested cities, and the prevalence of microsporidia ranged from 12.0 to $47.2 \%\left(X^{2}=38.376, \mathrm{df}=3, P<0.01\right)$ (Table $1)$. The Dutch breed (28.3\%) had a higher susceptibility to microsporidia infection than other breeds; however, the differences among pet rabbit breeds were not significant $\left(X^{2}=3.140, \mathrm{df}=5, P>0.05\right)$ (Table 2$)$. The prevalence in rabbits $\geq 12$ months of age was significantly

Table 1 The prevalence and genotype distribution of microsporidia in pet rabbits in southwestern China

\begin{tabular}{|c|c|c|c|c|c|c|c|c|}
\hline \multirow[t]{3}{*}{ Location } & \multirow{3}{*}{$\begin{array}{l}\text { No. positive/ } \\
\text { No. tested } \\
(\%)\end{array}$} & \multicolumn{7}{|c|}{ PCR positive } \\
\hline & & \multicolumn{2}{|c|}{ E. bieneusi } & \multicolumn{2}{|c|}{ E. cuniculi } & \multirow{2}{*}{$\begin{array}{l}\text { E. } \\
\text { intestinalis } \\
\text { No. (\%) }\end{array}$} & \multicolumn{2}{|c|}{ Mixed infection } \\
\hline & & No. (\%) & Genotype (n) & No. (\%) & $\begin{array}{l}\text { Genotype } \\
(n)\end{array}$ & & $\begin{array}{l}\text { No. } \\
(\%)\end{array}$ & Genotype (n) \\
\hline Chengdu & $70 / 313(22.4)$ & $\begin{array}{l}44 \\
(14.1)\end{array}$ & $\begin{array}{l}\text { SC02 (21), I (6), J (5), N, (8), SCR01 (1), } \\
\text { SCR02 (1), SCR06 (2) }\end{array}$ & $17(5.4)$ & | (11), || (6) & $7(2.2)$ & $2(0.6)$ & $\mathrm{SCO} 2+\mathrm{I}(1), \mathrm{J}+\mathrm{I}(1)$ \\
\hline Luzhou & $9 / 75(12.0)$ & $7(9.3)$ & $\operatorname{SCO} 2(3), I(3), J(1)$ & 0 & 0 & $2(2.7)$ & 0 & 0 \\
\hline Yaan & $50 / 106(47.2)$ & $\begin{array}{l}25 \\
(23.6)\end{array}$ & $\begin{array}{l}\mathrm{SCO} 2(8), \mathrm{I}(7), \mathrm{I}(5), \mathrm{N}(3), \mathrm{SCRO} 4(1) \\
\mathrm{CHY} 1 \text { (1) }\end{array}$ & $\begin{array}{l}15 \\
(14.2)\end{array}$ & $|(8)| \mid,(7)$ & $7(6.6)$ & $3(2.8)$ & $\mathrm{SCO} 2+\mathrm{I}(2), \mathrm{J}+\mathrm{II}(1)$ \\
\hline Ziyang & $16 / 90(17.8)$ & $\begin{array}{l}14 \\
(15.6)\end{array}$ & SC02 (7), I (2), SCR05 (2), SCR07 (3) & $2(2.2)$ & $\|(2)$ & 0 & 0 & 0 \\
\hline Total & $\begin{array}{l}145 / 584 \\
(24.8)\end{array}$ & $\begin{array}{l}90 \\
(15.4)\end{array}$ & $\begin{array}{l}\text { SC02 (39), I (21), N (13), J (6), CHY1 (1), } \\
\text { SCR01 (1), SCR02 (1), SCR04 (1), SCR05 (2), } \\
\text { SCR06 (2), SCR07 (3) }\end{array}$ & $34(5.8)$ & | (19), || (15) & $16(2.7)$ & $5(0.9)$ & $\begin{array}{l}\operatorname{SCO} 2+I(3), J+I I(1), J+I \\
(1)\end{array}$ \\
\hline
\end{tabular}


Table 2 The prevalence of microsporidia in pet rabbits by breed, age, and sex in southwestern China

\begin{tabular}{|c|c|c|c|c|c|}
\hline Group & No. of tested & No. of positive & Prevalence (\%) & $95 \% \mathrm{Cl}$ & $P$-value \\
\hline \multicolumn{6}{|l|}{ Breed } \\
\hline Dutch rabbit & 205 & 58 & 28.3 & $22.1-34.5$ & \multirow[t]{6}{*}{0.68} \\
\hline New Zealand Rabbit & 97 & 24 & 24.7 & $16.2-33.3$ & \\
\hline Lop ear rabbit & 94 & 20 & 21.3 & $13.0-29.6$ & \\
\hline Pygmy rabbit & 80 & 18 & 22.5 & $13.3-31.7$ & \\
\hline Pearl rabbit & 65 & 17 & 26.2 & $15.5-36.8$ & \\
\hline Lion head rabbit & 43 & 8 & 18.6 & $7.0-30.2$ & \\
\hline \multicolumn{6}{|l|}{ Age (months) } \\
\hline$\leq 6$ & 165 & 41 & 24.8 & $18.3-31.4$ & \multirow[t]{3}{*}{0.01} \\
\hline $6-12$ & 316 & 67 & 21.2 & $16.7-25.7$ & \\
\hline$\geq 12$ & 103 & 37 & 35.9 & $26.7-45.2$ & \\
\hline \multicolumn{6}{|l|}{ Sex } \\
\hline Male & 319 & 82 & 25.7 & $20.9-30.5$ & \multirow[t]{2}{*}{0.59} \\
\hline Female & 265 & 63 & 23.8 & $18.6-28.9$ & \\
\hline
\end{tabular}

higher $(35.9 \%)$ than those at 6-12 months of age $(21.2 \%)$ $\left(X^{2}=9.018, \mathrm{df}=2, P<0.05\right)$. However, there were no significant differences in the prevalence between males $(25.7 \%)$ and females $(23.8 \%)\left(\chi^{2}=0.289, \mathrm{df}=1, P>0.05\right)$.

\section{Molecular characterization of E. bieneusi and Encephalitozoon spp.}

Analysis of the ITS sequences of the E. bieneusi-positive samples revealed the presence of 11 distinct genotypes, including five known (SC02, I, N, J, CHY1) and six novel genotypes (SCR01, SCR02, SCR04 to SCR07). Genotype SC02 was the most prevalent $(43.3 \%, 39 / 90)$ and displayed $100 \%$ homology with the previously published GenBank Accession No. KY950533 (from a giant panda in China). Followed by genotype I (23.3\%, 21/90), which was identical to the sequence AF135836 (from cattle in Germany). Thirteen samples were characterized as genotype $\mathrm{N}$ and displayed $100 \%$ homology with the GenBank Accession No. AF267144, and six samples as genotype J identical to GenBank Accession No. AF135837. Genotype CHY1 showed $100 \%$ homology with the GenBank Accession No. KT267289 (from a yak in China).

For the novel genotypes of $E$. bieneusi, genotypes SCR01, SCR02 and SCR04 were found to have two, four, and five single nucleotides polymorphisms (SNPs), respectively, when compared to genotype SC02 (accession No. KY950533). Genotype SCR05 had six SNPs when compared to genotype XJH2 (accession No. KU194604; from a horse in China). Further, genotype SCR06 had seven SNPs in comparison to genotype J (GenBank Accession No. AF135837) and genotype SCR07 had eight SNPs when compared to genotype N (GenBank Accession No. AF267144). The genetic polymorphism of the novel genotypes within the $243 \mathrm{bp}$ of the ITS sequence are presented in Additional file 2.

Sequence analysis of 34 isolates of E. cuniculi revealed that 19 isolates were identical with genotype I (GenBank Accession No. KJ469979 from Gorilla beringei beringei in Rwanda) and 14 isolates were identical to genotype II (GenBank Accession No. GU213880 from a cat in Austria). The sequence of E. intestinalis showed 100\% identity with the deposited sequences in the GenBank (Accession No. GQ408912 from a patient with HIV in Russia).

Regarding to co-infections, three rabbits showed mixed infections of genotype $\mathrm{SC} 02$ of E. bieneusi and genotype I of E. cuniculi. One animal showed coinfections of genotype J of E. bieneusi and genotype II of E. cuniculi. Further, a co-infection of genotype J of $E$. bieneusi and genotype I of E. cuniculi was observed in one rabbit.

\section{Phylogenetic relationship of $E$. bieneusi}

The ITS sequence data for the 11 distinct genotypes identified in this study were included in a phylogenetic analysis, together with sequences representing 11 established groups of E. bieneusi. Five genotypes (SC02, SCR01, SCR02, SCR04, and SCR05) were classified into zoonotic potential group 1. Three known genotypes (I, $\mathrm{N}$, and J) were clustered into group2. Genotype SCR06 belonged to group 3. Genotypes CHY1 and SCR07 were classified into group 10 (Fig. 1).

\section{Discussion}

Although E. bieneusi and Encephalitozoon spp. mainly cause infections and potentially life-threatening diseases in individuals with immune deficiency, the routes of 


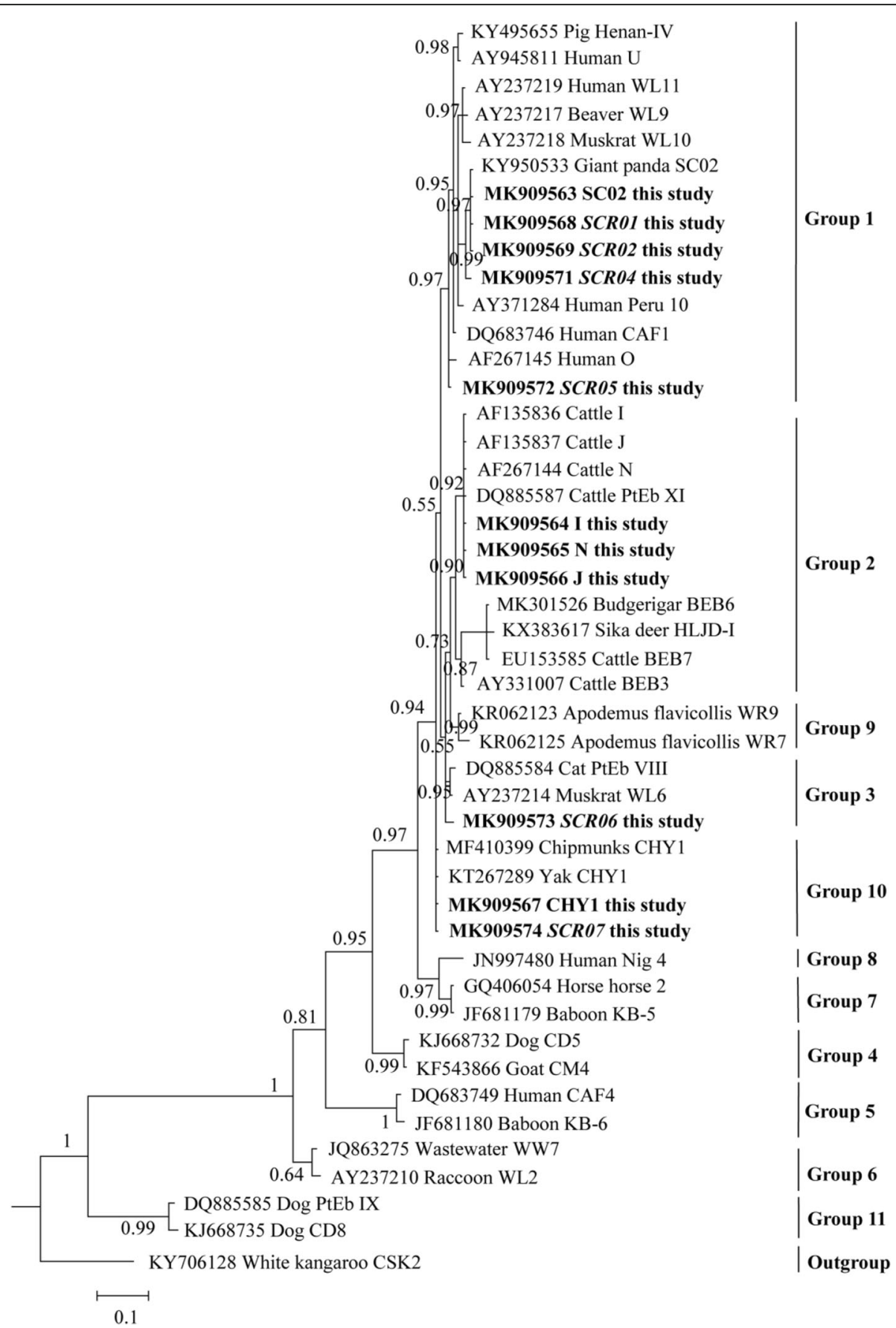

Fig. 1 Phylogenetic analysis of internal transcribed spacer of ribosomal RNA of E. bieneusi by Bayesian inference. Statistically significant posterior probabilities are indicated on the branches. Each sequence is identified by its accession number, host and genotype designation. Known and novel E. bieneusi genotypes identified in the present study are indicated by bold, and the novel genotypes are shown by italic. The E. bieneusi genotype CSK2 (KY706128) from white kangaroo was used as the outgroup

transmission as well as source of infection are not fully understood. Environmentally-resistant microsporidial spores of human and animal origin have been consistently found in surface waters, raising concerns about waterborne outbreaks [16]. So far, the increasing number of researchers have focused on animal microsporidiosis to explain possible sources of human microsporidiosis in the past few years, but few studies have been conducted on pet rabbits in close contact with humans. To the best of our knowledge, this is the first molecular identification of E. bieneusi and Encephalitozoon spp. in pet rabbits in China. 
In the present study, we found an age-related infection pattern, with animals $>1$ year being significantly more infected by microsporidia than younger ones $(x 2=9.018$, $\mathrm{df}=2, p<0.05$ ), which is consistent with previous findings in domestic rabbits in northeastern China [17]. In addition, similar findings were also demonstrated in other animals, such as dogs, donkeys, and foxes [18-20]. These findings might suggest that the parasite tends to accumulate with age and, therefore, could behave as a commensal rather than a pathogen.

E. bieneusi has been detected in a wide range of animals, such as macaques, pigs, cattle, horses, dogs, cats, raccoons, foxes, llamas, pigeons, and farmed rabbits [18, 21, 22]. The prevalence of E. bieneusi was $15.4 \%$ (90/ 584 ), which is similar with the previously reported in Rex rabbits $(14.7 \%, 22 / 150)$ and other pets in China [23-25], but higher than that most previously reported rates from Chinese provinces [26, 27] (Table 3). The differences in E. bieneusi infection rates may be due to the fact that rabbits are more susceptible to infection than dogs and cats. Genotype SC02 has been identified in a wide range of animals in China, including nonhuman primates, wild boars, horses, giant pandas, and squirrels [8]. Genotype $\mathrm{SCO} 2$ was predominant in the present study, which differs from the findings of previous studies in Rex rabbits in Heilongjiang province [23], and in domestic rabbits in Xinjiang [26]. Genotypes N, I, and J were originally detected in cattle, but recently these genotypes have also been detected in non-human primates, donkey, cats, sika deer, birds, and humans [21]. Genotype CHY1 is a synonym of genotype S7, which was identified in a patient in the Netherlands [33], as well as in yaks [34], in pet chipmunks [25], and more recently, in pet rats [35]. Notably, Cama et al. reported a possible transmission of $E$. bieneusi between children and guinea pigs in the same household, suggesting the possibility of zoonotic transmission between human and pet animal [36]. These data suggest that these genotypes have a broad host range and zoonotic potential.

Phylogenetic analysis was conducted to reveal the relationship and genetic diversity between the 11 genotypes identified in the present study and other representative known genotypes. The known genotype $\mathrm{SC} 02$ and four novel genotypes (SCR01, SCR02, SCR04, SCR05) belonged to group 1, which has been considered has significant zoonotic importance [3]. Group 1 consists of mostly genotypes from humans and genotypes from a wide range of animals, including nonhuman primates, porcines, bovines, cats, dogs, equines, carnivores, rodents, lagomorphs, marsupials, birds, and some rare hosts (e.g., bat, hippo, snake, muskrat, vole, beaver) [8, 37]. Although there is no clear evidence that human infection with $E$. bieneusi is related to pet rabbits, direct contact with infected rabbits or drinking contaminated water by spore of microsporidia is considered to be an important risk factor for the spread of microsporidiosis.

Table 3 The prevalence and genotypes of Enterocytozoon bieneusi in rabbits and various pets in China

\begin{tabular}{|c|c|c|c|c|}
\hline Province & Host & $\begin{array}{l}\text { No. positive/No. } \\
\text { examined (\%) }\end{array}$ & Genotype (n) & References \\
\hline Jilin & Rabbits & $3 / 174(1.7)$ & $\mathrm{D}(3)$ & {$[17]$} \\
\hline Liaoning & Rabbits & $1 / 136(0.7)$ & $\mathrm{D}(1)$ & {$[17]$} \\
\hline Heilongjiang & Rex rabbits & $22 / 150(14.7)$ & $\begin{array}{l}\text { CHN-RD1 (12), D (3), Type IV (2), I (1), Peru6 (1), CHN-RR1 (1), CHN-RR2 (1), CHN- } \\
\text { RR3 (1) }\end{array}$ & [23] \\
\hline Xinjiang & Rabbits & 9/321 (2.8) & J (5), BEB8 (3), Type IV (1) & [26] \\
\hline Jilin & Dog & $2 / 26(7.8)$ & CHN5 (1), CHN6 (1) & {$[28]$} \\
\hline Henan & Dog & $13 / 133(9.8)$ & PtEbIX (3), CM1 (2), D (2), Peru8 (1), type IV (1), CD2 (1), CD6 (1), CD7 (2) & {$[20]$} \\
\hline Shanxi & Dog & $6 / 30(20.0)$ & PtEbIX (2), EbpC (1), CD8 (2), CD9 (1) & [20] \\
\hline Chongqing & Dog & $4 / 34(11.8)$ & PtEbIX (2), CD8 (2) & [20] \\
\hline Heilongjiang & Dog & $18 / 267(6.7)$ & $\begin{array}{l}\text { D (1), EbpC (2), NED1 (1), NED2 (1); PtEb IX (14), NED3 (1), NED4 (1) (mix } \\
\text { infection) }\end{array}$ & [29] \\
\hline Shanghai & Dog & $29 / 485(6.0)$ & PtEb IX (28), D (1) & [30] \\
\hline Henan & Cat & $11 / 96(11.5)$ & D (3), BEB6 (2), I (1), PtEbIX (1), CC1 (1), CC2 (1), CC3 (1), CC4 (1) & [20] \\
\hline Heilongjiang & Cat & $3 / 52(5.8)$ & D (2), type IV (1) & [29] \\
\hline Shanghai & Cat & $9 / 160(5.6)$ & Type IV (5), D (4) & [30] \\
\hline Henan & Chinchilla & 4/102 (3.9) & $\mathrm{D}(2), \mathrm{BEB} 6(2)$ & [31] \\
\hline Beijing & Chinchilla & $1 / 26(3.8)$ & BEB6 (1) & [31] \\
\hline Sichuan & Pet birds & 97/387 (25.1) & D (41), SC02 (29), BEB6 (14), CHB1 (4), MJ5 (3), SCB-I (3), SCB-II (1), SCB-III (2) & {$[32]$} \\
\hline Sichuan & $\begin{array}{l}\text { Various pet } \\
\text { rabbits }\end{array}$ & $90 / 584(15.4)$ & $\begin{array}{l}\text { SC02 (39), I (21), N (13), J (6), CHY1 (1), SCR01 (1), SCR02 (1), SCR04 (1), SCR05 (2), } \\
\text { SCR06 (2), SCR07 (3) }\end{array}$ & This study \\
\hline
\end{tabular}


The prevalence of E. cuniculi in the present study was $5.8 \%$, which is lower than that in rabbits in Taiwan (67.8\%) [38], Italy (67.2\%) [39], and Austria (58.5\%) [40]. Based on the ITS region of rRNA gene, we identified genotypes I and II of E. cuniculi in pet rabbits. Type I primarily infects rabbits, and infections have been reported in the USA, Australia, and Europe [41]. Type II has only been confirmed in pigeons in Iran, in waters in Switzerland, and in pigs in Europe [42]. This is the first study of E. intestinalis infection in rabbits, reporting a prevalence of $2.7 \%$. Due to the lack of data regarding the prevalence of E. cuniculi and E. intestinalis in humans and other hosts in the investigated areas, the actual infection sources and transmission routes were not elucidated in the present study.

\section{Conclusions}

This is the first report of the E. bieneusi and Encephalitozoon spp. in pet rabbits in China. The overall prevalence of microsporidia in pet rabbits was $24.8 \%$ and some known zoonotic genotypes were identified, suggesting pet rabbits may play a role in the transmission of these pathogens to humans and other animals. These findings extend.

the knowledge of the microsporidia distribution among pet rabbits and provide fundamental data for controlling microsporidiosis in pet rabbits and humans.

\section{Methods}

\section{Collection of faecal samples}

Between July 2017 and January 2019, a total of 584 faecal samples were collected from rabbits in 12 pet shops located in four cities of Sichuan province, southwestern China. The pet shops were randomly selected according to the estimated number of pet shops per area. All tested pet shops only raised rabbits and served as suppliers of rabbits to other pet shops. Before signing a formal consent, the manager of each pet shop was informed about the study purpose and procedures. Only one sample was collected from each animal. Faecal samples were collected from the bottom of each cage and then individually placed into $30 \mathrm{~mL}$ sterile plastic containers with ice packs. All samples were transported to the laboratory within $24 \mathrm{~h}$. The age, sex, source, and health condition of each rabbits were recorded at the sampling site. All animals were healthy and none showed any clinical signs of gastrointestinal disease at the time of sampling.

\section{DNA extraction}

All faecal samples were suspended in distilled water, and the suspension was then passed through a $250 \mu \mathrm{m}$ pore size wire mesh sieve. The filtrate was centrifuged at
$1500 \times \mathrm{g}$ for $10 \mathrm{~min}$. Genomic DNA was extracted from $\sim 200 \mathrm{mg}$ of each processed faecal sample using QIAamp ${ }^{\star}$ Stool Mini Kit (Qiagen, Hilden, Germany) according to the manufacturer's instructions. DNA was eluted in $50 \mu \mathrm{l}$ of nuclease-free water (Promega, Madison, USA), and acquired DNA was stored at $-20^{\circ} \mathrm{C}$ until use.

\section{PCR amplification and sequence analysis}

E. bieneusi genotypes were determined by nested PCR of the ITS region of rRNA as described by Sulaiman et al. [43]. Encephalitozoon spp. ITS was amplified using MSP-1 and MSP-2A as the outer primer pair, and MSP-3 and MSP-4A as the inner primer pair [44, 45]. PCR amplification primers and cycling conditions in this study are presented in Additional file 1. PCR was performed with a $50 \mu \mathrm{l}$ volume containing $25 \mu \mathrm{l}$ Taq PCR Master Mix (Sangon Biotech Co., Ltd., Shanghai, China), $2 \mu \mathrm{l}$ each primer $(0.4 \mu \mathrm{M}), 1 \mu \mathrm{l}$ of each DNA sample, $1.5 \mu \mathrm{l} \quad \mathrm{MgCl}_{2} \quad(25 \mathrm{mM})$ and nuclease-free water up to desired volume. Positive and negative controls were included in all the PCR tests. The secondary PCR products were examined by $2 \%$ agarose gel electrophoresis and visualized after ethidium bromide staining.

Positive secondary PCR products were sent to Life Technologies for sequencing with an ABI 3730 DNA Analyzer using the BigDye ${ }^{\circledR}$ Terminator v3.1 cycle sequencing kit (Applied Biosystems, Foster City, CA, USA) at the Sangon Biotech Company (Chengdu, China). The sequences were edited and aligned using ClustalW (http://www.ebi.ac.uk/Tools/msa/clustalw2/) and compared with reference sequences from GenBank using BLAST tool (http://blast.ncbi.nlm.nih.gov/Blast.cgi). The accuracy of the sequences of the novel genotypes was confirmed by resequencing the obtained amplicons.

\section{Phylogenetic and statistical analyses}

Bayesian inference (BI) and Monte Carlo Markov Chain (MCMC) methods were used to construct the phylogenetic tree in MrBayes version 3.2.5 [46]. Akaike Information Criteria (AIC) test in ModelFinder was used for evaluating the substitution model that best fit the dataset [47]. The posterior probability (pp) values were calculated by running 2,000,000 generations. A 50\% majorityrule consensus tree was constructed from the final $75 \%$ of the trees generated by BI. Analyses were run three times to ensure convergence and insensitivity to priors.

Data were analyzed using SPSS statistical software (version 22) and the Chi-square test was used to detect significant differences. $P$-values $<0.05$ were considered statistically significant. 


\section{Supplementary information}

Supplementary information accompanies this paper at https://doi.org/10. 1186/s12917-020-02434-z.

Additional file 1: Table S1. Primer sequences, fragment lengths and annealing temperatures used in this study.

Additional file 2: Figure S1. Sequence variation in the ITS region of the rRNA gene of Enterocytozoon bieneusi isolates from pet rabbits. The ITS sequences of five known genotypes (SCO2, I, N, J, and CHY1) and the six novel genotypes (SCR01, SCR02, SCR04 to SCR07), identified in this study, were aligned with each other.

\section{Abbreviation}

ITS: Internal transcribed spacer; E. bieneusi: Enterocytozoon bieneusi; $E$. cuniculi: Encephalitozoon cuniculi; E. intestinalis: Encephalitozoon intestinalis; E. hellem: Encephalitozoon hellem; NIH: National Institutes of Health; EPA: Environmental Protection Agency; rRNA: Ribosomal RNA; BI: Bayesian inference; MCMC: Monte Carlo Markov Chain; AIC: Akaike Information Criteria; pp.: Posterior probability

\section{Acknowledgements}

We would like to thank Xueping Zhang and Xuehan Liu for comments on the draft manuscript.

\section{Authors' contributions}

LD designed the project, performed experiments and discussed the data. YC performed the experiments, and analyzed the data. LX analyzed and discussed the data. WW collected the faecal samples. Z-Y Z collected the faecal samples. HL collected the faecal samples. Z-J Z designed the project. HF analyzed and discussed the data. GP conceptualized and approved the manuscript. All authors read and approved the final version of the manuscript.

\section{Funding}

The study was financially supported by the National Science and Technology Department "13th five-year" Special Subproject of China (No. 2016YFD0501009) and the Chengdu Giant Panda Breeding Research Foundation (CPF2017-12, CPF2015-09, CPF2015-07). The funding bodies had no role in designing the study, sample collection, analysis, and interpretation of data or in writing the manuscript. Lei Deng was the recipient of scholarships from the Chinese Scholarship Council (CSC).

\section{Availability of data and materials}

Representative nucleotide sequences were deposited in GenBank with the following accession numbers: MK909563- MK909569 and MK909571MK909574 (E. bieneusi), MK909577 (E. intestinalis), MK909562 and MN749308 (E. cuniculi).

\section{Ethics approval and consent to participate}

This study complied with the guidelines of the Regulations for the Administration of Affairs Concerning Experimental Animals and was approved by the Animal Ethical Committee of Sichuan Agricultural University. No animals were harmed during the sampling process. The written permission was obtained from the managers of the pet shops before the faecal samples were collected.

\section{Consent for publication}

Not applicable.

\section{Competing interests}

The authors declare that they have no competing interests.

Received: 19 March 2020 Accepted: 16 June 2020

Published online: 22 June 2020

\section{References}

1. Didier ES, Weiss LM. Microsporidiosis. current status. Cur Opin Infect Dis. 2006;19(5):485

2. Patrick K. Five questions about microsporidia. Plos Pathogens. 2009:5(9): e1000489.
3. Li W, Xiao L. Multilocus sequence typing and population genetic analysis of Enterocytozoon bieneusi: host specificity and its impacts on public health. Front Genet. 2019;10:307.

4. Santín M, Fayer R. Microsporidiosis. Enterocytozoon bieneusi in domesticated and wild animals. Res Vet Sci. 2011;90(3):363-71.

5. Kašičková $D$, Sak $B$, Kváč $M$, Ditrich $O$. Sources of potentially infectious human microsporidia. Molecular characterisation of microsporidia isolates from exotic birds in the Czech Republic, prevalence study and importance of birds in epidemiology of the human microsporidial infections. Vet Parasitol. 2009;165(1):125-30.

6. Espern A, Morio F, Miegeville M, Illa H, Abdoulaye M, Meyssonnier V, et al. Molecular study of microsporidiosis due to Enterocytozoon bieneusi and Encephalitozoon intestinalis among human immunodeficiency virus-infected patients from two geographical areas: Niamey, Niger, and Hanoi, Vietnam. J Clin Microbiol. 2007:45(9):2999-3002.

7. Zhang Y, Koehler AV, Wang T, Haydon SR, Gasser RB. New operational taxonomic units of Enterocytozoon in three marsupial species. Parasit Vectors. 2018;11(1):371

8. $\quad$ Li W, Feng Y, Santin M. Host specificity of Enterocytozoon bieneusi and public health implications. Trends Parasitol. 2019;35(6):436-51.

9. Zhang Y, Koehler AV, Wang T, Haydon SR, Gasser RB. First detection and genetic characterisation of Enterocytozoon bieneusi in wild deer in Melbourne's water catchments in Australia. Parasit Vectors. 2018;11(1):2.

10. Didier ES, Baker CRVD, Rogers LB, Bertucci DC, Shadduck JA. Identification and characterization of three Encephalitozoon cuniculi strains. Parasitol. 1995; 111(4):411-21.

11. Milnes E, Delnatte P, Cai HY, Nemeth N. Systemic Encephalitozoonosis due to Encephalitozoon cuniculi strain IV in a vancouver island marmot (Marmota vancouverensis). J Zoo Wildl Med. 2018;49(2):484

12. Mathis A, Tanner I, Weber R, Deplazes P. Genetic and phenotypic intraspecific variation in the microsporidian Encephalitozoon hellem. Int J Parasitol. 1999:29(5):767.

13. Qiu L, Xia W, Li W, Ping J, Ding S, Liu H. The prevalence of microsporidia in China: a systematic review and meta-analysis. Sci Rep. 2019;9(1):3174.

14. Wang S, Yao Z, Li L, Pan Y, Li P, Nan X, Xie Q, Zhang Z. Seroprevalence of Toxoplasma gondii and Encephalitozoon cuniculi among domestic rabbits in central China. Parasite. 2018;25(3):9.

15. Yang Z, Yang F, Wang J, Cao J, Zhao W, Gong B, Yan J, Zhang W, Liu A Shen $Y$. Multilocus sequence typing and population genetic structure of Cryptosporidium cuniculus in rabbits in Heilongjiang Province, China. Infect Gene Evol. 2018;64:249-53.

16. Didier ES, Stovall ME, Green LC, Brindley PJ, Sestak K, Didier PJ. Epidemiology of microsporidiosis: sources and modes of transmission. Vet Parasitol. 2004;126(1-2):145-66.

17. Zhang XX, Jing J, Cai YN, Wang CF, Peng X, Yang GL, Quan Z. Molecular characterization of Enterocytozoon bieneusi in domestic rabbits (Oryctolagus cuniculus) in northeastern China. Korean J Parasitol. 2016;54(1):81-5.

18. Yue DM, Ma JG, Li FC, Hou JL, Zheng WB, Zhao Q, Zhang XX, Zhu XQ. Occurrence of Enterocytozoon bieneusi in Donkeys (Equus asinus) in China: A Public Health Concern. Front Microbiol. 2017;8(641).

19. Zhang XX, Cong W, Lou ZL, Ma JG, Zheng WB, Yao QX, Zhao Q, Zhu XQ. Prevalence, risk factors and multilocus genotyping of Enterocytozoon bieneusi in farmed foxes (Vulpes lagopus), Northern China. Parasites Vectors. 2016;9(1):1-7.

20. Karim MR, Dong H, Yu F, Jian F, Zhang L, Wang R, Zhang S, Rume Fl, Ning C, Xiao L. Genetic diversity in Enterocytozoon bieneusi isolates from dogs and cats in China: host specificity and public health implications. J Clin Microbiol. 2014;52(9):3297-302.

21. Wang SS, Wang RJ, Fan XC, Liu TL, Zhang LX, Zhao GH. Prevalence and genotypes of Enterocytozoon bieneusi in China. Acta Trop. 2018:183.

22. Li DF, Zhang $Y$, Jiang YX, Xing JM, Tao DY, Zhao AY, Cui ZH, Jing B, Qi M, Zhang LX. Genotyping and zoonotic potential of Enterocytozoon bieneusi in pigs in Xinjiang, China. Front Microbiol. 2019;10:2401.

23. Yang Z, Zhao W, Shen Y, Zhang W, Shi Y, Ren G, et al. Subtyping of Cryptosporidium cuniculus and genotyping of Enterocytozoon bieneusi in rabbits in two farms in Heilongjiang Province, China. Parasite. 2016;23(52).

24. Deng L, Li W, Yu X, Gong C, Liu X, Zhong Z, et al. First report of the humanpathogenic Enterocytozoon bieneusi from red-bellied tree squirrels (Callosciurus erythraeus) in Sichuan, China. PloS One. 2016;11(9):e0163605.

25. Deng L, Li W, Zhong Z, Chai Y, Yang L, Zheng H, Wang W, Fu H, He M, Huang $X$. Molecular characterization and new genotypes of Enterocytozoon 
bieneusi in pet chipmunks (Eutamias asiaticus) in Sichuan province, China. BMC Microbiol. 2018;18(1):37.

26. Zhang $X$, Qi M, Jing B, Yu F, Wu Y, Chang Y, et al. Molecular Characterization of Cryptosporidium spp., Giardia duodenalis, and Enterocytozoon bieneusi in Rabbits in Xinjiang, China. J Eukaryot Microbiol. 2018

27. Galván-Díaz AL, Magnet A, Fenoy S, Henriques-Gil N, Haro M, Gordo FP, et al. Microsporidia Detection and Genotyping Study of Human Pathogenic E. bieneusi in Animals from Spain. PloS One 2014, 20;9(3):e92289.

28. Zhang $X$, Wang $Z$, Su $Y$, Liang $X$, Sun $X$, Peng $S$, et al. Identification and genotyping of Enterocytozoon bieneusi in China. J Clin Microbiol. 2011;49(5): 2006-8.

29. Li W, Li Y, Song M, Lu Y, Yang J, Tao W, et al. Prevalence and genetic characteristics of Cryptosporidium, Enterocytozoon bieneusi and Giardia duodenalis in cats and dogs in Heilongjiang province, China. Vet Parasitol. 2015;208(3-4):125-34.

30. Xu H, Jin Y, Wu W, Li P, Wang L, Li N, Feng Y, Xiao L. Genotypes of Cryptosporidium spp., Enterocytozoon bieneusi and Giardia duodenalis in dogs and cats in Shanghai, China. Parasit Vectors. 2016;9:121.

31. Qi M, Luo N, Wang H, Yu F, Wang R, Huang J, Zhang L. Zoonotic Cryptosporidium spp. and Enterocytozoon bieneusi in pet chinchillas (Chinchilla lanigera) in China. Parasitol Intern. 2015;64(5):339-41.

32. Deng L, Yue CJ, Chai YJ, Wang WY, Su XY, Zhou ZY, et al. New genotypes and molecular characterization of Enterocytozoon bieneusi in pet birds in southwestern China. IJP. 2019;10:164-9.

33. Ten Hove RJ, Van Lieshout L, Beadsworth MB, Perez MA, Spee K, Claas EC, Verweij JJ. Characterization of genotypes of Enterocytozoon bieneusi in immunosuppressed and immunocompetent patient groups. J Eukaryot Microbiol. 2009;56(4):388-93.

34. Li J, Qi M, Chang Y, Wang R, Li T, Dong H, Zhang L. Molecular characterization of Cryptosporidium spp., Giardia duodenalis, and Enterocytozoon bieneusi in captive wildlife at Zhengzhou zoo, China. J Eukaryot Microbiol. 2015;62(6):833-9.

35. Wang J, LV C, Zhao D, Zhu R, Li C, Qian W. First detection and genotyping of Enterocytozoon bieneusi in pet fancy rats (Rattus norvegicus) and Guinea pigs (Cavia porcellus) in China. Parasite. 2020;27:21.

36. Cama VA, Pearson J, Cabrera L, Pacheco L, Gilman R, Meyer S, Ortega Y, Xiao L. Transmission of Enterocytozoon bieneusi between a child and Guinea pigs. J Clin Microbiol. 2007;45(8):2708-10.

37. Zhao A, Li D, Wei Z, Zhang Y, Peng Y, Zhu Y, Qi M, Zhang L. Molecular detection and genotyping of Enterocytozoon bieneusi in racehorses in China. Front Microbiol. 2019:10:1920.

38. Tee KY, Kao JP, Chiu HY, Chang MH, Wang JH, Tung KC, et al. Serological survey for antibodies to Encephalitozoon cuniculi in rabbits in Taiwan. Vet Parasitol. 2011;183(1-2):68-71.

39. Dipineto L, Rinaldi L, Santaniello A, Sensale M, Cuomo A, Calabria M, et al. Serological survey for antibodies to Encephalitozoon cuniculi in pet rabbits in Italy. Zoonoses Public Health. 2010;55(3):173-5.

40. Gruber A, Pakozdy A, Weissenböck H, Csokai J, Künzel F. A retrospective study of neurological disease in 118 rabbits. J Comp Pathol. 2009;140(1):31-7.

41. Thomas C, Finn M, Twigg L, Deplazes P, Thompson RC. Microsporidia (Encephalitozoon cuniculi) in wild rabbits in Australia. Australian Vet J. 2010 75(11):808-10

42. Němejc K, Sak B, Květoňová D, Hanzal V, Janiszewski P, Forejtek P, et al. Prevalence and diversity of Encephalitozoon spp. and Enterocytozoon bieneusi in wild boars (Sus scrofa) in Central Europe. Parasitol Res. 2014;113(2):761.

43. Sulaiman IM, Fayer R, Lal AA, Trout JM, Rd SF, Xiao L. Molecular Characterization of Microsporidia Indicates that Wild Mammals Harbor HostAdapted Enterocytozoon spp. as well as Human-Pathogenic Enterocytozoon bieneusi. Appl Environ Microbiol. 2003;69(8):4495.

44. Franzen C, Müller A. Molecular techniques for detection, species differentiation, and phylogenetic analysis of microsporidia. Clin Microbiol. 1999;12(2):243-85.

45. Katzwinkel-Wladarsch S, Lieb M, Helse W, Löscher T, Rinder H. Direct amplification and species determination of microsporidian DNA from stool specimens. Trop Med Int Heal. 1996;1(3):373-8.

46. Huelsenbeck JP, Ronquist F. MRBAYES: Bayesian inference of phylogenetic trees. Bioinformatics. 2001;17(8):754-5.

47. Kalyaanamoorthy S, Minh BQ, Tkf W, Von HA, Jermiin LS. ModelFinder: fast model selection for accurate phylogenetic estimates. Nature Methods. 2017 14(6).

\section{Publisher's Note}

Springer Nature remains neutral with regard to jurisdictional claims in published maps and institutional affiliations.
Ready to submit your research? Choose BMC and benefit from:

- fast, convenient online submission

- thorough peer review by experienced researchers in your field

- rapid publication on acceptance

- support for research data, including large and complex data types

- gold Open Access which fosters wider collaboration and increased citations

- maximum visibility for your research: over $100 \mathrm{M}$ website views per year

At BMC, research is always in progress.

Learn more biomedcentral.com/submissions 